\section{Polycaprolactone nanofiber mesh with adhered liposomes as a simple delivery system for bioactive growth factors}

\author{
Andrea Staffa, Karolina Vocetkova, \\ Vera Sovkova, Michala Rampichova, \\ Eva Filova, Evzen Amler \\ Institute of Experimental Medicine of \\ the Czech Academy of Sciences, Prague, \\ Czech Republic
}

\begin{abstract}
Polycaprolactone (PCL) nanofibers were prepared using electrospinning and functionalized with liposomes containing growth factors by simple surface adhesion. A tight interaction between the liposomes and PCL nanofibers has been clearly demonstrated. Additionally, the interaction of the liposomes with the PCL nanofibers was visualized by FESEM. The potential of the liposome-immobilized scaffold as a delivery system for synthetic growth factors, and as a suitable system for mesenchymal stem cells (MSCs) adhesion and proliferation, was evaluated by confocal microscopy, DNA synthesis rate and dsDNA amount determination. The results showed that the growth factors adhered to the surface of the PCL nanofibers stimulated cell proliferation mainly up to day 7 , and that afterwards their effect was significantly lower. By contrast, the release of growth factors from liposomes resulted in gradual proliferation of MSCs throughout the whole experiment. Moreover, the functionalized nanofibers stimulated type II collagen production, which was confirmed by immunohistochemical staining using monoclonal antibody against type II collagen. The study has indicated that growth factor-enriched liposomes adhered to the PCL nanofiber system could be useful as a drug delivery tool in various biomedical applications.
\end{abstract}

\section{Introduction}

Nanofibers prepared by the electrospinning technique have been tested as a drug delivery system in wide number of studies. Electrospun nanofiber surfaces have been functionalized to achieve sustained delivery of various bioactive substances, just by physical adsorption. ${ }^{1}$ The main consideration for the development of the successful delivery systems for tissue engineering are the bioactivity of the biomolecules adsorbed on the scaffolds and the controlled release of these biomolecules according to the time frame of tissue regeneration. ${ }^{2} \mathrm{Nie}$ et al. ${ }^{3}$ loaded bone morphogenetic protein-2 (BMP-2) into electrospun PLGA scaffolds by dipping the scaffold into an aqueous phase containing biomolecules. They showed that BMP-2 adsorbed to the PLGA scaffolds reached over $75 \%$ release within 5 days and almost complete release within 20 days. ${ }^{3}$ Simple physical adsorption of proteins on nanofiber surfaces has been therefore seldom used, due to the uncontrolled release profiles. $^{2}$ Moreover, the surface characteristics such as hydrophilicity/hydrophobicity arising from the chemical composition of the nanofibers may not be satisfactory for inducing selective cell adhesion, migration, and proliferation. ${ }^{4}$ The surface chemistry can be modified for optimal tissue engineering by cold plasma treatments, surface graft polymerization, UV-ozone treatments, wet chemical methods and by co-electrospinning of bioactive agents and polymers. ${ }^{1,5-7}$ Naturally derived biomolecules and specific cell ligands on nanofiber surfaces were immobilized to modulate the cell-matrix interactions, thus achieving improved cell adhesion, proliferation and differentiation. ${ }^{8}$ Functionalized nanofibers have also been prepared by adhesion of bioactive factorloaded micro/nanoparticles, e.g. lipid or polymeric particles. ${ }^{9,1}$ Immobilized nanoparticles interacted with the targeted cells and the nanofiber scaffold, on which the cells were cultured, thus creating a combined sustained and local release system. ${ }^{11}$ The nanoparticles outperformed the conventional delivery of free drugs in terms of delivery of an encapsulated drug and its sustained release.

Liposomes are widely used nanoparticles and carrier systems for delivery of hydrophilic or hydrophobic biomolecules, drugs, small interference RNA (siRNA), viruses and bacteria etc.. ${ }^{12-14}$ Liposomes were already used as a simple drug delivery system in combination with electrospun fibers in our laboratory. ${ }^{15}$ Unilamellar liposomes containing foetal bovine serum (FBS) were adhered to polyhydroxyethylmethacrylate (pHEMA) electrospun fibers. Liposomes adhered to the nanofibers released FBS and supported cell adhesion, their further proliferation and, moreover, chondrogenic differentiation.

The aim of the current study was to develop a combined release system that would protect and deliver labile synthetic growth factors and/or other therapeutically active compounds to the intended site of injury. By delivering the bioactive compounds enclosed in liposomes, their presence in the site of injury should be extended
Correspondence: Andrea Staffa, Institute of Experimental Medicine, The Czech Academy of Sciences, Videnska 1083, 14220 Prague, Czech Republic.

Tel.: +420241062763.

E-mail: andrea_mickova@labdemo.cz

Key words: Polycaprolactone nanofiber; Adhered liposomes; Simple delivery system; Bioactive growth factors.

Acknowledgements: This work was supported by project LO1508 from the Ministry of Education, Youth and Sports of the Czech Republic. Special thanks should be given to Matej Buzgo for his valuable support and constructive recommendations on this project.

Received for publication: 20 March 2017.

Revision received: 3 May 2017.

Accepted for publication: 20 MAy 2017.

This work is licensed under a Creative Commons Attribution 4.0 License (by-nc 4.0).

CCopyright A. Mickova et al., 2017

Licensee PAGEPress, Italy

Translational Medicine Reports 2017; 1:6716 doi:10.4081/tmr.6716

and their therapeutic effect prolonged. The proposed system was tested in vitro using porcine mesenchymal stem cells.

\section{Materials and Methods}

\section{Needleless electrospinning for pro- duction of nanofibers}

$24 \%(\mathrm{w} / \mathrm{v})$ solutions of polycaprolactone (PCL) with a molecular weight (MW PCL) of 45000 (Sigma-Aldrich, MO, USA) dissolved in chloroform:ethanol (8:2) were prepared for liposome and growth factor adhesion studies. Electrospinning was carried out on a Nanospider ${ }^{\mathrm{TM}}$ device (Elmarco, Czech Republic) as described previously in detail. ${ }^{16} \mathrm{~A}$ high-voltage source generated voltages of up to $56 \mathrm{kV}$, and the polymer solutions were connected to the high-voltage electrode. The electrospun nanofibers were deposited on a grounded collector electrode. The distance between the tip of the syringe needle and the collecting plate was $12 \mathrm{~cm}$. All electrospinning processes were performed at room temperature $\left(\mathrm{RT} ; \sim 24^{\circ} \mathrm{C}\right.$ ) and relative humidity of $\sim 50 \%$.

\section{Preparation of liposomes}

A polydisperse suspension of multilamellar liposomes was prepared from soybean-derived L- $\alpha$-phosphatidylcholine (PC; Avanti Polar Lipids, Inc., AL, USA) using the extrusion method. ${ }^{17}$ Briefly, $25 \mathrm{mg}$ of 
soybean PC was dissolved in chloroform (1 $\mathrm{mL}$ ); to prepare fluorescently labelled liposomes, FITC - labelled phosphatidylcholine (FITC-PC: PC from soybean in a 1:1,000 molar ratio; FLL; Molecular Probes, OR, USA) was added and subsequently evaporated under a flow of $\mathrm{N}_{2}$ at $4^{\circ} \mathrm{C}$ to form a thin lipid film. The dried lipid films prepared from PC were then resuspended in $1 \mathrm{~mL}$ of Tris-buffered saline (TBS; $150 \mathrm{mM} \mathrm{NaCl}$ and $50 \mathrm{mM}$ Tris, $\mathrm{pH}$ 7.4) for the preparation of liposomes without growth factors, as controls (LC). For fluorescent spectroscopy measurements the lipid films were resuspended in a 8Aminonaphthalene-1,3,6-trisulfonic acid (ANTS) $+p$-xylene-bis-pyridinium bromide (DPX) system (1.5 mL of solution containing $15 \mathrm{mM}$ ANTS, $45 \mathrm{mM}$ DPX in TBS, $\mathrm{pH}=7.4$; Molecular Probe, OR, USA). Liposomes with the encapsulated substances were prepared by resuspending the dry lipid films in $1 \mathrm{~mL}$ of TBS with a mixture of recombinant growth factors $(0.1$ $\mu \mathrm{g} / \mathrm{mL}$ transforming growth factor beta 1 (TGF- $\beta 1) ; 10 \mu \mathrm{g} / \mathrm{mL}$ insulin-like growth factor 1 (IGF-1); $5 \mu \mathrm{g} / \mathrm{mL}$ basic fibroblast growth factor (bFGF) in TBS. To obtain liposomes of uniform size, the multilamellar liposomes were extruded several times through polycarbonate filters with a welldefined pore-size ( $1 \mu \mathrm{m}$ diameter) using an Avanti Mini-extruder (Avanti Polar Lipids Inc., AL, USA). Unencapsulated FITC-PC and ANTS + DPX system was separated on a Sephadex G-25 column (Sigma-Aldrich, MO, USA). Unencapsulated mixture of the growth factors was separated from the liposomes using a Sephacryl HR-500 column (GE Healthcare, IL, USA).

\section{Measurement of liposome size}

The average size distribution of the liposomes was measured using dynamic light scattering on Mastersizer 3000 (model MAZ3000, Malvern Instruments Ltd, UK). $1 \mathrm{~mL}$ of the sample was applied to wet dispersion unit and DLS of 5 measurements was performed using both blue and red laser. The size was calculated using model for spherical particles. Results were given in average mean diameters reported as function of the size distribution and intensity.

\section{Scaffold composite preparation}

PCL nanofibers were cut into round patches of $6 \mathrm{~mm}$ diameter and sterilized using ethylene oxide at $37^{\circ} \mathrm{C}$. For the in vitro study, the composite scaffolds composed of PCL nanofibers and the recombinant growth factor mixture (GFM), the liposomes with encapsulated mixture of recombinant growth factors (LGFM) and the liposomes with encapsulated TBS buffer [served as control (LC)], were prepared by
2 hour incubation of the PCL nanofibers in $6 \mathrm{~mL}$ of the respective solutions. To prepare the composite scaffolds for fluorescence studies, the PCL nanofibers were immersed into $1 \mathrm{~mL}$ of the solution containing fluorescently labelled liposomes (FLL) overnight, subsequently the non-adhered liposomes were removed by intensive washing with TBS buffer; or the PCL nanofibers were immersed into the liposome solution of ANTS + DPX for $60 \mathrm{sec}-$ onds and subsequently rinsed with TBS ( $\mathrm{pH}$ 7.4) overnight. After the incubation, the scaffolds were rinsed with TBS repeatedly to remove the non-adhered GFM, LGFM, LC and FLL.

\section{Fluorescent spectroscopy measure- ments of liposome-nanofiber inter- actions}

Nanofibers with liposomes containing ANTS + DPX (rinsed and washed with TBS solution overnight) were subsequently dissolved in ethanol to release the ANTS + DPX system and treated with Triton X-100 afterwards. Fluorescence measurements were performed as described in Fiser and Konopasek $^{18}$ on a FluoroMax-3 JY-Horiba spectrofluorimeter (Horiba Scientific, NJ, USA). Excitation and emission wavelengths were 370 and $505 \mathrm{~nm}$, respectively (both with bandwidths of $4 \mathrm{~nm}$ ). A suspension $(1.5 \mathrm{~mL})$ was placed into a $1 \times 1 \mathrm{~cm}$ quartz cuvette, and the recorded fluorescence intensities were corrected for the background (vesicles without ANTS and DPX, about $2 \%$ of the total fluorescence intensity) and for the effect of dilution due to the addition of DPX and Triton X-100.

\section{Cryo-field emission scanning elec- tron microscopy}

Cryo-field emission scanning electron microscopy (cryo-FESEM) was used to visualize the liposomes adhered to the PCL nanofibers. Samples were prepared using the Quorum Technologies device PP3010T (Quorum Technologies Ltd, UK). Briefly, the samples were frozen rapidly in liquid $\mathrm{N}_{2}$ $\left(-210^{\circ} \mathrm{C}\right)$ and then transferred to the cryostage of the preparation chamber (PP3010T) of a FEI Nova NanoSem 450 (FEI, Czech Republic). Samples were freeze-fractured at $-90^{\circ} \mathrm{C}$ and subsequently coated with $5 \mathrm{~nm}$ of platinum. After the preparation, the samples were examined in the frozen state at a temperature of approximately $-175{ }^{\circ} \mathrm{C}$ on the FEI Nova NanoSEM 450 microscope, using TLD detector and CBS at $5 \mathrm{kV}$.

\section{Confocal laser scanning microscopy analyses}

FLL adhered to the PCL nanofibers, prepared for the fluorescence study as described in the Scaffold Composite Preparation section, were visualized using a LSM 5 DUO (FITC fluorescence, $\lambda_{\mathrm{ex}}=488$ $\left.\mathrm{nm}, \lambda_{\mathrm{em}}=505-550 \mathrm{~nm}\right)$ confocal laser scanning microscope (Zeiss, Germany).

\section{Mesenchymal stem cells isolation, culture, and seeding on the function- alized nanofiber scaffolds}

Bone marrow was aspirated from the iliac wing of a minipig into a $5 \mathrm{~mL}$-syringe containing $1 \mathrm{~mL}$ of PBS and $25 \mathrm{IU}$ heparin under general anaesthesia. The mononuclear cells were isolated using gradient separation with the plasma substitute Gelofusine ${ }^{\circledR}$. Briefly, 4-5 mL of the bone marrow mixture was mixed with $1.5 \mathrm{~mL}$ Gelofusine ${ }^{\circledR}$. After $30 \mathrm{~min}$ of sedimentation, the upper and medium layers containing plasma, mononuclear cells, and erythrocytes were aspirated, and centrifuged at 270 $\times g$ for $15 \mathrm{~min}$. Subsequently, the medium layer with mononuclear cells was aspirated and seeded in tissue culture flasks. Adherent cells were cultured in Minimum Essential Medium (MEM) (with L-glutamine, PAA Laboratories, UK) containing 10\% fetal bovine serum (Mycoplex, PAA Laboratories, Austria), $100 \mathrm{IU} / \mathrm{mL}$ penicillin, and $100 \mu \mathrm{g} / \mathrm{mL}$ streptomycin. The cells were passaged using the trypsinEDTA method before confluence was reached. The cells from the second or third passage were used for the cell culture study. The PCL scaffolds with adhered GFM, LGFM and LC were prepared as described in the Scaffold composite preparation section. The scaffolds were seeded with mesenchymal stem cells (MSCs; $37 \times 10^{3}$ cells $/ \mathrm{cm}^{2}$ ) and cultured in $200 \mu \mathrm{L}$ of MEM (Sigma-Aldrich, MO, USA), 1\% fetal bovine serum, and penicillin/streptomycin for 14 days. The DNA content was determined on day $1,3,7$, and 14 , the DNA synthesis rate on day 3 and 7, and the immunofluorescence staining analysis for type II collagen staining was performed on day 7 , and 14 .

\section{dsDNA content measurement}

The dsDNA content of the MSCs cultured on the scaffolds with adhered GFM, LGFM and LC was determined using the Quant-iT ${ }^{\mathrm{TM}}$ PicoGreen ${ }^{\circledR}$ Assay Kit (Invitrogen Ltd., CS, USA) on day 1, 3, 7, and 14. To perform the analysis, $250 \mu \mathrm{L}$ of a cell lysis buffer $(0.2 \%(\mathrm{v} / \mathrm{v})$ Triton X-100, $10 \mathrm{mM}$ Tris (pH 7.0) (1 mM EDTA) was added to each sample. Subsequently, the samples underwent a total number of 3 freeze/thaw cycles (the samples were firstly frozen at $-80^{\circ} \mathrm{C}$ and then thawed at RT) in total, to prepare a cell lysate. In between the 
freeze/thaw cycles, the samples were roughly vortexed. A calibration curve was constructed using the standards supplied in the kit to calculate the dsDNA content on the scaffolds. Samples were loaded in triplicates and the fluorescence intensity was measured on a multiplate fluorescence reader (Synergy HT, Biotek, VT, USA; $\lambda_{\text {ex }}=$ $480-500 \mathrm{~nm}, \lambda_{\text {em }}=520-540 \mathrm{~nm}$ ).

\section{Evaluation of DNA synthesis rate}

The DNA synthesis rate of the MSCs seeded on the functionalized nanofibrous scaffolds was determined using a colorimetric immunoassay based on the measurement of 5-bromo-2"'-deoxyuridine (BrdU) incorporation during the DNA synthesis (Cell proliferation, BrdU, colorimetric; Roche Diagnostics $\mathrm{GmbH}$, Germany). The assay was performed according to the manufacturer's instructions. Briefly, on days 3 and 7, $120 \mu \mathrm{L}$ BrdU labeling solution was added to each well containing a scaffold. BrdU was allowed to incorporate into the cells in a $\mathrm{CO}_{2}$ incubator at $37^{\circ} \mathrm{C}$ for 2 hours. Subsequently, the supernatant was removed and the scaffolds were incubated with FixDenat solution, to fix the cells and denature the DNA at RT for $30 \mathrm{~min}$. The supernatant was once again removed and $100 \mu \mathrm{L}$ of anti-BrdU-peroxidase (1:100 dilutions) was added and kept at RT for 60 min. After removal of the unbound antibody conjugate, $100 \mu \mathrm{L}$ of a substrate solution was added (6 min incubation) and the reaction was completed by addition of $25 \mu \mathrm{L}$ of $\mathrm{H}_{2} \mathrm{SO}_{4}$ solution $(1 \mathrm{M})$. Then, $100 \mu \mathrm{L}$ of the solution was transferred to a 96-well plate and measured within $5 \mathrm{~min}$ at $450 \mathrm{~nm}$ with a reference wavelength of $690 \mathrm{~nm}$ using an ELISA plate reader (EL 800; BioTek, VT, USA). The blank corresponded to scaffolds without cells, with or without BrdU.

\section{Fluorescence confocal microscopy of mesenchymal stem cells on the func- tionalized nanofiber scaffolds}

DiOC6 (3,3'-diethyloxacarbocyanine iodide) and propidium iodide (PI) were used to visualize adhesion of MSCs to the scaffolds 24 hours after the cell seeding. Samples were fixed with frozen methanol ($20{ }^{\circ} \mathrm{C}$ ), rinsed twice with PBS, incubated in $10 \mu \mathrm{g} / \mathrm{mL}$ DiOC6 (Invitrogen, CA, USA) for $45 \mathrm{~min}$ at RT, and then incubated in 5 $\mu \mathrm{g} / \mathrm{mL}$ propidium iodide (Sigma-Aldrich, MO, USA) in PBS for $10 \mathrm{~min}$. The scaffolds were rinsed twice with PBS and scanned. A Zeiss LSM 5 DUO confocal microscope; $\lambda_{\mathrm{ex}}=488$ and $560 \mathrm{~nm}$ and $\lambda_{\mathrm{em}}=$ 505-550 and 575-650 nm was used for DiOC6 and propidium iodide detection, respectively. DiOC6 staining was used to visualize biomembranes (green colour), while propidium iodide staining was used to visualize cell nuclei (red color).

\section{Detection of chondrogenic marker using indirect immunofluorescence staining}

The presence of type II collagen as a marker of chondrogenic differentiation was visualized using indirect immunofluorescence staining on day 7 and 14 as described previously. Briefly, the samples were fixed with $10 \%$ formaldehyde/PBS for $10 \mathrm{~min}$ and permeabilized by PBS with $1 \%$ BSA $/ 0.1 \%$ Triton $\mathrm{X}-100$ for $30 \mathrm{~min}$ at RT. The samples were incubated with the primary antibody against type II collagen (clone II-II6B3, 1:20 dilution, 1 hour incubation at RT; Developmental Studies Hybridoma Bank, created by the NICHD of the NIH and maintained at The University of Iowa, Department of Biology, Iowa City, IA 52242). Following three washes with PBS $/ 0.05 \%$ Tween 20, the samples were incubated with a secondary antibody (Alexa Fluor 488-conjugated goat anti-mouse IgG $(\mathrm{H}+\mathrm{L}), 1: 300$ dilution, 45 min incubation at RT; Invitrogen, CA, USA). The cell nuclei were counterstained with propidium iodide (15 min incubation at RT) and subsequently washed three times with PBS $/ 0.05 \%$ Tween 20. The stained samples were visualized using a Zeiss LSM 5 DUO confocal microscope; $\lambda_{\mathrm{ex}}=488$ and $560 \mathrm{~nm}$ and $\lambda_{\mathrm{em}}=505-$ 550 and $575-650 \mathrm{~nm}$ was used for Alexa Fluor 488 and propidium iodide detection, respectively.

\section{Intensity measurement}

The mean intensity of images was calculated by ImageJ software using raw images. The mean grey level of 16-bit images of newly formed type II collagen was analyzed by software. The intensity refers to particular area of cells and does not reflect the overall intensity on the scaffold; therefore it is a semi-quantitative value.

\section{Statistical analysis}

The results were evaluated statistically using One Way Analysis of the Variance (ANOVA) and Student-Newman-Keuls method. The level of significance was set at 0.05 . The data are presented as the mean value $\pm \mathrm{SD}$ (standard deviation).

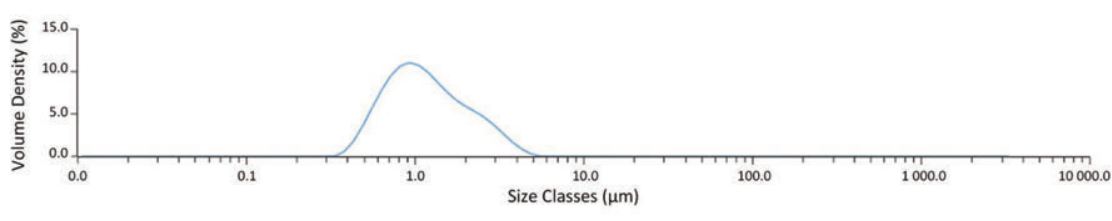

Figure 1. Liposome size measurement characterized by dynamic light scattering.

\section{Results}

\section{Interaction of liposomes with the surface of polycaprolactone nanofiber scaffolds}

Liposomes may be combined with nanofiber scaffolds to promote local and sustained delivery of loaded bioactive agents. PCL nanofibers prepared by needleless electrospinning technique were chosen as a model for functionalization by liposomes. Stereological measurements of the PCL fibrous layers identified a population of nano-/microfibers with a minimum of non-fibrous defects. The mean diameter of the thin nanofibrous fraction was $385 \pm 102$ $\mathrm{nm}$. In addition, a less abundant microfiber fraction was detected with a mean diameter of $2832 \pm 1821 \mathrm{~nm}$. Pore distribution of the nanofibrous scaffold showed mean diameter of $2.1 \pm 1.4 \mu \mathrm{m}$ with the largest pores about $10 \mu \mathrm{m}$.

The liposomes were prepared from a fluorescent phospholipid mixture (FITCPC:PC in a 1:1000 molar ratio). The size distribution of the liposomes was characterized by dynamic light scattering measurements. The obtained data showed that the most represented size of the liposomes was

The average size distribution of the liposomes was measured using dynamic light scattering on Mastersizer 3000 (model MAZ3000, Malvern Instruments Ltd).

Functionalization of the PCL nanofibers was achieved by incubation with FLL. To visualize the interactions of FLL with the PCL nanofiber mesh, confocal microscopy (Figure 2) was applied. The large surface area of the PCL nanofibers resulted in the immobilization of the liposomes on the surface of the PCL nanofiber mesh. Adhesion of the liposomes was also confirmed quantitatively by fluorescence measurements of liposomes containing the DPX + ANTS system. Approximately $29 \%$ of the liposomes remained tightly bound to the nanofibers after they had been rinsed and washed with the TBS solution overnight.

Additionally, cryo-FESEM was applied to visualize the interactions of liposomes with the PCL nanofibers. Tight interaction of the liposomes with the PCL nanofibers approximately $1 \mu \mathrm{m}$ (Figure 1). 
was observed (Figure 3).

Cryo-FESEM showed the liposomes adhered to the PCL nanofibers (liposomes are indicated by arrows).

\section{In vitro testing of the functionalized nanofibers}

The prepared composite scaffolds were tested as a possible drug delivery system for TGF- $\beta 1$, bFGF and IGF- 1 . The mixture of the growth factors was either simply adhered to the surface of the PCL nanofibrous scaffold (GFM), or it was encapsulated into the liposomes (LGFM). To increase the effect of the growth factors, the cells were cultured under serum-reduced conditions $(1 \% \mathrm{FBS})$ and the medium was not refreshed.

The number of the cells on the scaffolds was quantified using a dsDNA-specific assay on days 1, 3, 7 and 14 (Figure 4A). The data showed improved initial adhesion of the cells on the control sample (LC) on day 1 . It resulted in a statistically significant increase in the DNA content on the control sample on day 3, when compared to the GFM and LGFM samples. On day 7, the number of the cells on the GFM scaffolds was significantly higher than on the LGFM and the control samples. Interestingly, the concentration of DNA in the LGFM samples was significantly higher on day 14 , in comparison to the GFM and LC samples. Data suggested that the free growth factors on GFM scaffolds enhanced the proliferation of MSCs in the 1\% FBS medium from the early stages of the experiment until the

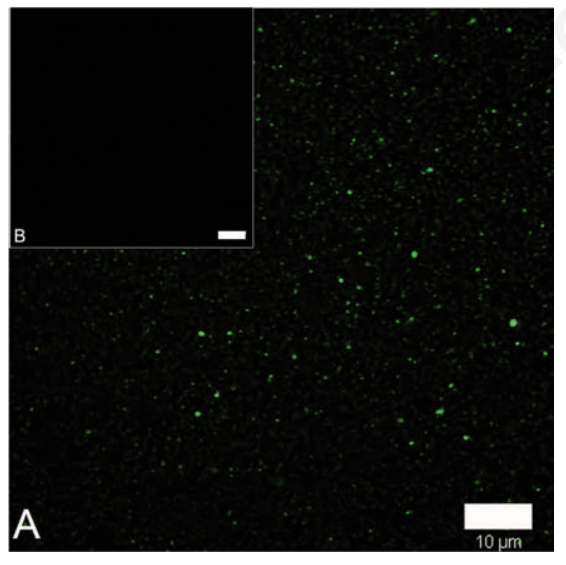

Figure 2. Confocal microscopy of fluorescently labelled liposomes immobilized to polycaprolactone nanofiber mesh. (A) Visualization of fluorescently labelled liposomes prepared by the addition of fluorescein isothiocyanate-labelled phosphatidylcholine to $\mathrm{L}-\alpha$-phosphatidylcholine from soybeans by confocal microscopy. (B) Pure polycaprolactone nanofiber mesh without liposomes as a control. Scale bars indicate (A, B) $10 \mu \mathrm{m}$. day 7. In comparison to that, the release of growth factors from the liposomes was slower. It is assumed, that the sustained release of the growth factors from the liposome gradually enhanced the proliferation of MSCs on the LGFM scaffolds throughout the culture.

DNA synthesis rate on the scaffolds was examined using a BrdU assay on day 3 and 7 (Figure 4B). The BrdU assay showed significantly higher DNA synthesis rate of MSCs on the GFM scaffolds compared to the LGFM and LC scaffolds on day 3, indicating positive effect of the high concentration of the growth factors in the GFM samples in the early stages of the experiment. On day 7, significant differences in the DNA synthesis rate were detected on the LC scaffolds in comparison to the GFM scaffold.

The cell adhesion on the GFM, LGFM and LC scaffolds was visualized using confocal microscopy on day 1 (Figure 5A-C). The cell membranes were stained with DiOC6 (green) and the nuclei were counterstained using propidium iodide (red). The cells were well spread and were randomly distributed on the GFM and LGFM scaffolds. More pronounced adhesion of the cells was observed on the LC scaffolds.

.

Figure 3. Cryo-field emission scann electron microscopy of immobilized liposomes on polycaprolactone nanofibers.

Table 1. Semi-quantitative analysis of type II collagen production on growth factors, growth factors embedded in liposomes and control scaffolds.

\begin{tabular}{lccc} 
Sample type & Area & Mean intensity & Standard deviation \\
GFM - day 7 collagen type II & 763872 & 12.785 & 17.521 \\
GFM - day 14 collagen type II & 1309878 & 5.003 & 13.221 \\
\hline LGFM - day 7 collagen type II & 630427 & 21.619 & 32.689 \\
LGMF - day 14 collagen type II & 799750 & 8.683 & 15.848 \\
\hline LC - day 7 collagen type II & 767376 & 3.514 & 8.893 \\
LC day 14 collagen type II & 1279112 & 5.648 & 13.062 \\
\hline
\end{tabular}

GFM, growth factors; LGFM, growth factors embedded in liposomes; LC, controls.
A

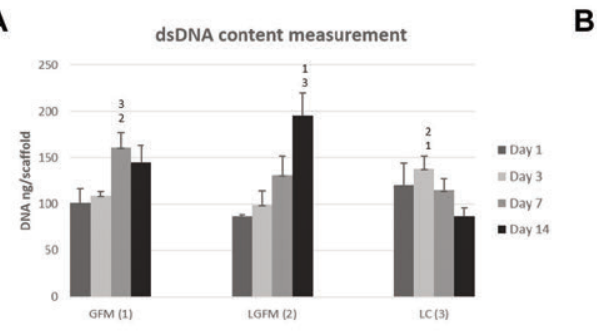

These findings support the obtained data from the Picogreen and BrdU assays.

In order to visualize the matrix synthesis, immunohistochemical staining against type II collagen was performed on day 7 (Figure 5D-F) and on day 14 (Figure 5G-I). On day 7, larger amount of the newly formed type II collagen was shown to be produced by groups of cells on the LGFM samples as well as on GFM samples compared to LC samples. Semi-quantitative analyses showed no significant differences of type II collagen production on GFM and LGFM samples (Table 1). Only a small amount of type II collagen was detected on

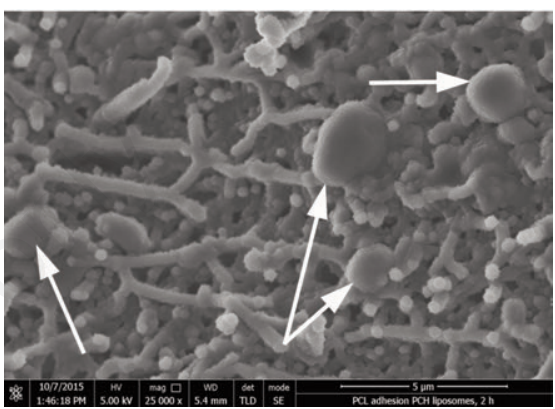

Figure 4. Determination of dsDNA content using the PicoGreen method and DNA synthesis rate using the BrdU assay. Mesenchymal stem cells were cultured on the polycaprolactone scaffolds functionalized with the growth factors and the growth factors embedded in liposomes. Liposomes with embedded Tris-buffered saline adhered on the polycaprolactone fibers (controls) were used as a control. (A) DNA content was determined on day $1,3,7$ and 14. The level of statistical significance for the assay is denoted above the bars $(P<0.05)$. (B) DNA synthesis rate was determined on day 3 and 7 . The level of statistical significance for the assay is denoted above the bars $(\mathrm{P}<0.05)$. 
the control samples, which was also indicated by the intensity measurement of green channel on LC scaffolds on day 7 (Figure $5 \mathrm{~F}$ and Table 1) and on day 14 (Figure 5I and Table 1). Moreover, smaller aggregates of cells were detected in the LC samples. The production of the type II collagen on all scaffolds was lower on day 14 compared to day 7 , due to the unfavourable environment created by the supplementation of the cell culture medium with 1\% FBS (i.e. 10 times lower FBS concentration than in a standard cell culture).

\section{Discussion}

PCL nanofibers were used as a system suitable for functionalization with liposomes. A tight interaction between the liposomes and the PCL nanofibers was clearly demonstrated by cryo-FESEM. Quantitative analysis showed that approximately $29 \%$ of the liposomes remained tightly attached to the nanofiber mesh after being rinsed with TBS overnight. The results were in accordance with the results of Rampichova et al. reflected the large surface-to-volume ratio of the nanofibers. ${ }^{15}$ Nanofibers are extensively proposed for tissue engineering applications, and also as drug delivery systems. ${ }^{5,6}$

It is necessary to develop an effective drug delivery system to protect the labile synthetic growth factors and to extend their presence at the site of injury. ${ }^{19}$ Functionalized PCL nanofibrous scaffolds were prepared and tested as a possible drug delivery system for various bioactive compounds. TGF- $\beta 1$, bFGF and IGF- 1 have been shown to increase MSCs proliferation..$^{20}$ In the current study, the mixture of the growth factors and also growth

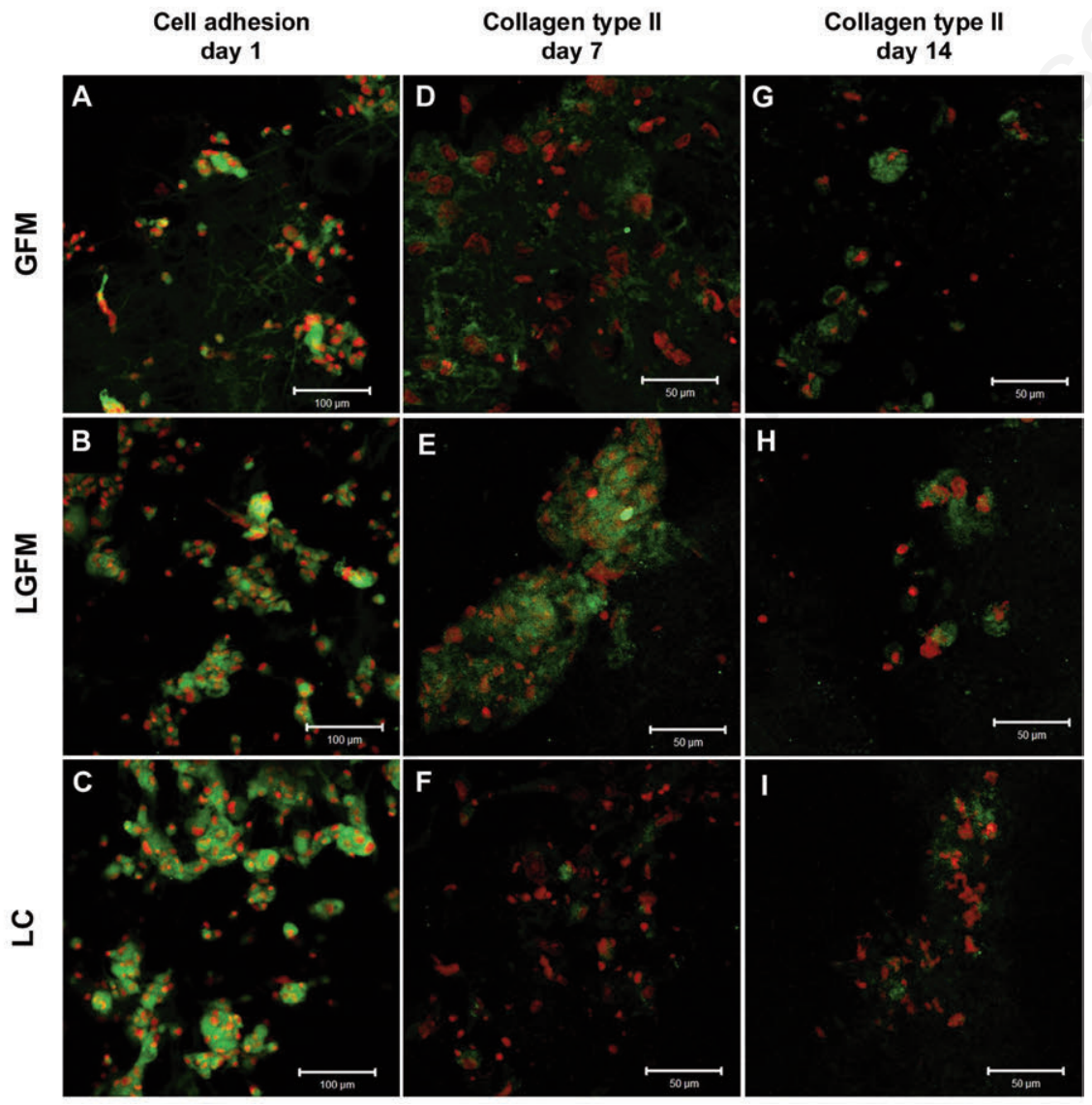

Figure 5. Confocal microscopy of mesenchymal stem cells on polycaprolactone functionalized scaffolds. Adhesion of the cells on day 1 to (A) growth factors scaffolds, (B) growth factors embedded in liposomes scaffolds, (C) control scaffolds. The nuclei were stained with propidium iodide (red) and the membranes were stained with DiOC6 (green). Synthesis of type II collagen on day 7 on (D) growth factors, (E) growth factors embedded in liposomes and (F) control scaffolds. Synthesis of type II collagen on day 14 on (G) growth factors, (H) growth factors embedded in liposomes, (I) control scaffolds. Type II collagen was visualized by immunofluorescence (green) and the cell nuclei were stained with propidium iodide (red). The scale bars indicate (A-C) $100 \mu \mathrm{m}$ and (D-I) $50 \mu \mathrm{m}$. factors incorporated into liposomes were immobilized to the PCL nanofiber mesh and subsequently seeded with MSCs. Adhesion and proliferation of the seeded MSCs on the functionalized scaffolds was confirmed by confocal microscopy, BrdU DNA synthesis rate assay, and dsDNA content measurement via the Picogreen assay. The cells were cultured in a medium supplemented with $1 \% \mathrm{FBS}$, and in order to preserve the release of the stimulating factors, the medium was not refreshed during the cell culture.

On all tested scaffold, we have observed good cell adhesion on day 1. Adhesion of GFM or LGFM to the PCL nanofiber scaffolds in the same location as the MSCs led to the localization of synthetic growth factors in the vicinity of the cells. On the LGFM scaffold, DNA amount was increasing till the day 14 along with the highest DNA amount on day 14, which can be explained by sustained release of growth factors from the liposomes to the neighbouring cells during the entire culture period. We have observed even slower sustained release of growth factors from liposomes embedded in core-shell nanofibers. ${ }^{21}$ Other possible explanation of the sustained release from liposomes is interactions between the phospholipids and the growth factors. Wiessner and Hwang 22 reported interaction between insulin and phosphatidylcholine bilayer. In other experiments, we have observed significantly increased absolute release of insulin from nanofibers from polyvinylalcohol containing liposomes and subsequently immersed into solution containing growth factors. ${ }^{23}$ Oppositely, on the GFM scaffolds, the higher BrdU incorporation on day 3, and higher DNA content on day 7 was probably caused by high growth factor concentration at the beginning of the experiment. As expected, the degradation of growth factors in the medium slowed down the cell growth on the GFM scaffolds. The growth of MSCs is affected by their environment as well as by growth factors, cytokines or proteins of extracellular matrix which they synthesize. Growth factors or cytokines subsequently have paracrine or autocrine effects on cells. ${ }^{24}$ We have seeded relatively high density of MSCs on the scaffolds, which positively influenced their survival in a medium supplemented with only $1 \%$ FBS by their own growth factor synthesis. However, their proliferation in medium with decreased growth factors and FBS is lower compared to MSCs in the medium with growth factors. Thus, the proliferation of cells on LC scaffolds was prolonged to day 7, when higher BrdU incorporation compared to GFM scaffolds was found. 
Various in vitro studies have demonstrated the effects of IGF-1, TGF- $\beta 1$ and bFGF in stimulating the production of chondrocyte specific extracellular matrix (ECM). ${ }^{25,26}$ Immunohistochemical staining of the type II collagen was used to confirm the induction of the chondrocyte-specific ECM production. The marker protein was produced on GFM and LGFM scaffolds. In addition, it has been observed that cell differentiation was accompanied by decreased cell proliferation. ${ }^{27} \mathrm{We}$ had similar observation in chondrogenic differentiation of MSCs (data not shown). The results indicated that the growth factors stimulated qualitatively superior matrix production and thus induced differentiation of the MSCs even in $1 \%$ FBS. The advantage of the LGFM was the slower and sustained release of the embedded growth factors compared to the GFM.

\section{Conclusions}

High levels of free growth factors are correlated with an increased risk of cancer. The possible use of their lower concentrations and the protection of the growth factors from the surrounding environment could minimize any potential risks. A disadvantage of the conventional liposomes is their short-term retention and fast washout from the site of application. The combined liposome-nanofiber scaffold could overcome the obstacles of conventional liposomes, and could serve as a local delivery system for susceptible bioactive molecules in various biomedical applications. However, in order to optimize the system it will be necessary to carry out a more complex study, showing in detail the influence of the dosage and the release kinetics of the growth factors from the liposomes adhered to the nanofibers on MSC viability, proliferation and differentiation.

\section{References}

1. Mattanavee W, Suwantong O, Puthong $\mathrm{S}$, et al. Immobilization of biomolecules on the surface of electrospun polycaprolactone fibrous scaffolds for tissue engineering. ACS Appl Mater Interfaces 2009;1:1076-85.

2. Ji W, Sun Y, Yang F, et al. Bioactive electrospun scaffolds delivering growth factors and genes for tissue engineering applications. Pharm Res 2011;28:125972.

3. Nie H, Soh BW, Fu YC, Wang CH.
Three-dimensional fibrous PLGA/HAp composite scaffold for BMP-2 delivery. Biotechnol Bioeng 2008;99:223-34.

4. Chung HJ, Park TG. Surface engineered and drug releasing pre-fabricated scaffolds for tissue engineering. Adv Drug Deliver Rev 2007;59:249-62.

5. Dahlin RL, Kasper FK, Mikos AG. Polymeric nanofibers in tissue engineering. Tissue Eng Part B Rev 2011;17:349-64.

6. Darain F, Chan WY, Chian KS. Performance of surface-modified polycaprolactone on growth factor binding, release, and proliferation of smooth muscle cells. Soft Mater 2011;9:64-78.

7. He W, Yong T, Teo WE, et al. Fabrication and endothelialization of collagen-blended biodegradable polymer nanofibers: potential vascular graft for blood vessel tissue engineering. Tissue Eng 2005;11:1574-88.

8. Wang YY, Lu LX, Feng ZQ, et al. Cellular compatibility of RGD-modified chitosan nanofibers with aligned or random orientation. Biomed Mater Engl 2010;5:054112.

9. Lima AC, Sher P, Mano JF. Production methodologies of polymeric and hydrogel particles for drug delivery applications. Expert Opin Drug Del 2012;9: 231-48.

10. Ruggeri F, Akesson A, Chapuis P-Y, et al. The dendrimer impact on vesicles can be tuned based on the lipid bilayer charge and the presence of albumin. Soft Mater 2013;9:8862-70.

11. Santo VE, Gomes ME, Mano JF, Reis RL. From nano- to macro-scale: nanotechnology approaches for spatially controlled delivery of bioactive factors for bone and cartilage engineering. Nanomedicine (Lond) 2012;7:1045-66.

12. Ignatius R, Mahnke K, Rivera M, et al. Presentation of proteins encapsulated in sterically stabilized liposomes by dendritic cells initiates CD8+ T-cell responses in vivo. Blood 2000,96:350513.

13. Lowery A, Onishko H, Hallahan DE, Han Z. Tumor-targeted delivery of liposome-encapsulated doxorubicin by use of a peptide that selectively binds to irradiated tumors. J Contr Rel 2011; 150:117-24.

14. Mufamadi MS, Choonara YE, Kumar P, et al. Ligand-functionalized nanoliposomes for targeted delivery of galantamine. Int J Pharm 2013;448:267-81.

15. Rampichova M, Martinova L, Kostakova E, et al. A simple drug anchoring microfiber scaffold for chon- drocyte seeding and proliferation. J Mat Sci-Mat 2012;23:555-63.

16. Lukas D, Sarkar A, Martinová L, et al. Physical principles of electrospinning (Electrospinning as a nano-scale technology of the twenty-first century). Textile Progress 2009;41:59-140.

17. Mayer LD, Bally MB, Hope MJ, Cullis PR. Techniques for encapsulating bioactive agents into liposomes. Chem Phys Lipids 1986;40:333-45.

18. Fiser R, Konopasek I. Different modes of membrane permeabilization by two RTX toxins: HlyA from Escherichia coli and CyaA from Bordetella pertussis. Biochim Biophys Acta 2009;1788: 1249-54.

19. Demidova-Rice TN, Hamblin MR, Herman IM. Acute and impaired wound healing: pathophysiology and current methods for drug delivery. Part 2: role of growth factors in normal and pathological wound healing: therapeutic potential and methods of delivery. In: Advances of skin wound care. United States, 2012, 25, pp. 349-70.

20. Shanmugarajan TS, Kim B-S, Lee H, Im G-I. Growth factors and signaling pathways in the chondrogenic differentiation of mesenchymal stem cells. Tissue Eng Regen Med 2011;8:292-9.

21. Mickova A, Buzgo M, Benada O, et al. Core/shell nanofibers with embedded liposomes as a drug delivery system. Biomacromolecules 2012;13:952-62.

22. Wiessner JH, Hwang KJ., K.J. Binding of insulin to the external surface of liposomes. Effect of surface curvature, temperature and lipid composition. Biochim Biophys Acta 1982;689:490-8.

23. Filová E, Rampichová M, Litvinec A, et al. A cell-free nanofiber composite scaffold regenerated osteochondral defects in miniature pigs. Int J Pharm 2013; 447:139-49.

24. Baraniak PR, McDevitt TC. Stem cell paracrine actions and tissue regeneration. Regen Med 2010;5:121-43.

25. Yu D-A, Han Y, Kim B-S. Stimulation of chondrogenic differentiation of mesenchymal stem cells. Int J Stem Cells 2012;5:16-22.

26. Luyten FP. Cartilage-derived morphogenetic proteins - Key regulators in chondrocyte differentiation? Acta Orth Scan 1995;66:51-4.

27. Ruijtenberg S, Van Den Heuvel S. Coordinating cell proliferation and differentiation: Antagonism between cell cycle regulators and cell type-specific gene expression. Cell Cycle 2016;5: 196-212. 